\title{
¿LA TEORÍA ENNOBLECE LA PRÁCTICA? SOBRE HACIA LA CIENCIA DEL LENGUAJE. EJERCICIOS DE ANÁLISIS GRAMATICAL (1916), DE RITA PÉREZ DE BERTELLI
}

\author{
Esteban Lidgett \\ Universidad de Buenos Aires (Argentina) \\ elidgett@filo.uba.ar \\ Guillermo Toscano y García \\ Universidad de Buenos Aires (Argentina) \\ gtoscano@filo.uba.ar
}

Recibido: 04/06/2020 - Aprobado: 04/08/2020 - Publicado: 15/04/2021

DOI: doi.org/10.17533/udea.lyl.n79a16

\begin{abstract}
Resumen: En 1916, Rita Pérez de Bertelli publicó Hacia la ciencia del lenguaje. Ejercicios de análisis gramatical, texto que, acompañando el impulso reformador que el normalismo venía pregonando para la educación primaria, aspiraba a renovar la enseñanza escolar de la gramática en la Argentina. Este trabajo propone abordar la gramática de la autora, con el objetivo de analizar el desarrollo de su propuesta didáctica en respuesta a los modelos tradicionales de enseñanza que serían ampliamente cuestionados por el normalismo durante las primeras décadas del siglo xx.
\end{abstract}

Palabras clave: gramática escolar; Argentina; siglo xx; Rita Pérez de Bertelli; historiografía lingüística.

\section{DOES THEORY ENNOBLES PRACTICE? ON HACIA LA CIENCIA DEL LENGUAJE. EJERCICIOS DE ANÁLISIS GRAMATICAL (1916), BY RITA PÉREZ DE BERTELLI}

\begin{abstract}
In 1916, Rita Pérez de Bertelli published Hacia la ciencia del lenguaje. Ejercicios de análisis gramatical, a text that, while accompanying the reformist impulse that teaching schools had been proclaiming for elementary education, aspired to renew the learning of grammar in Argentinian schools. This work proposes to address the author's grammar with the aim of analyzing the development of its didactic proposal in response to the traditional models of teaching that would be widely questioned by teaching schools during the first decades of the 20th century.
\end{abstract}

Key words: school grammar; Argentina; 20th century; Rita Pérez de Bertelli; linguistic historiography. 


\section{Introducción}

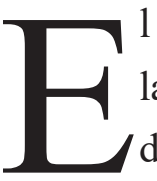

1 desarrollo de la investigación grupal «Los inicios de la gramática escolar en la Argentina (1863-1922)», en la que se inscribe este trabajo, así como la culminación de la investigación precedente, «La configuración

de la gramática escolar argentina (1862-1922)», ha propiciado en los últimos años la publicación de una serie de trabajos que, desde una perspectiva centrada en la historiografía lingüística (Koerner, 2007; Swiggers, 2009), o más específicamente, en la denominada historia de la gramaticografía didáctica (Swiggers, 2012), han procurado abordar la corriente gramatical escolar desarrollada en Argentina entre fines del siglo XIX y comienzos del xx. El extenso trabajo documental desarrollado en el marco de estas investigaciones permitió reconstruir por primera vez un corpus de más de 300 obras publicadas en dicho período y, en consecuencia, habilitó la posibilidad de que nuevas investigaciones historiográficas focalizaran su atención en la descripción de un conjunto de textos mayormente desconocido hasta entonces. Así, un primer grupo de trabajos, continuadores de la línea teórica propuesta inicialmente para la gramaticografía local por los estudios señeros de Calero Vaquera (2008 y 2009) y García Folgado (2010), ha centrado su atención en la caracterización de obras o autores particulares, generalmente de la segunda mitad del siglo XIX (Toscano y García \& García Folgado, 2015; Lidgett, 2011 y 2018; Lidgett \& Toscano y García, 2020). Un segundo grupo de investigaciones, por su parte, ha asumido una perspectiva más global sobre la selección de las obras, centrando su abordaje en un período determinado o en un conjunto de gramáticas (Gaviño Rodríguez, 2017; Lidgett 2017a, 2017b; Toscano y García \& García Folgado, 2012).

No obstante, son aún escasos los trabajos que ocupan, más específicamente, de obras publicadas durante las primeras décadas del siglo anterior (Lacanna, 2011; Toscano y García \& García Folgado, 2017), momento en que tanto el desarrollo del sistema escolar a nivel nacional como el crecimiento de la industria editorial impulsaron notablemente el volumen y la variedad del corpus gramatical ${ }^{1}$. Con el objetivo de avanzar en la caracterización de la gramática escolar de esta etapa, el presente trabajo propone un abordaje de la obra Hacia la ciencia del lenguaje. Ejercicios de análisis gramatical, de Rita Pérez de Bertelli. Publicada en 1916, en un contexto en que el normalismo ha consolidado su impulso renovador en la escuela primaria y en la formación de maestros, es una gramática escrita para la escuela normal que, como muchas otras del período, aspira a renovar las estrategias didácticas para la enseñanza de la lengua materna en las escuelas.

Sin embargo, dicha gramática muestra una serie de rasgos peculiares que la destacan en el corpus de la gramática escolar argentina. Por un lado, se trata de una de las pocas gramáticas escrita por mujeres ${ }^{2}$, en un contexto en que el mercado editorial de las gramáticas estaba previsiblemente dominado por varones. En efecto, dentro del conjunto de textos de la gramática escolar argentina, publicado entre 1817 y 1922 , se han registrado 13 gramáticas escritas por mujeres sobre un total de 315 obras. Si se considera el número de autores, de los 99 que 1. De acuerdo con los datos relevados en el proyecto marco de este trabajo, entre 1894 y 1922 la cantidad de gramáticas publicadas casi duplica a la de la etapa anterior: se publican 175 gramáticas en esta etapa, mientras que entre 1866 y 1893 , se registran 94 publicaciones (Toscano y García, Lidgett \& García Folgado, 2019).

2. Véase Barcia (2004), para la gramática de Hernández (1878), posiblemente la primera del género. 
Hacia la ciencia del lenguaje. Ejercicios de análisis gramatical (1916), de Rita Pérez de Bertelli conforman la selección de obras, solo 8 son mujeres ${ }^{3}$. Estos guarismos evidencian que, aunque la participación de la mujer en el ámbito educativo en general distaba de ser escasa, sobre todo tras el impulso que recibe la formación de maestras en el contexto de la emergencia del normalismo, su intervención en el mercado editorial vinculado al sistema educativo seguía siendo marginal ${ }^{4}$.

Por otro lado, la gramática de Pérez de Bertelli plantea un desarrollo didáctico que, si bien reconoce sus antecedentes en la tradición normalista durante el siglo anterior, lo hace en el contexto de lo que se propone como una actualización de la propuesta educativa de Amadeo Jacques, figura destacada en el proceso de organización de la enseñanza nacional en 1863, cuyo influjo se pudo registrar también en otras gramáticas del corpus escolar argentino, como las de Pujolle (1867) o Wernicke (1867). Esta actualización se despliega a partir de un recorrido que aspira a que los estudiantes adquieran los contenidos teóricos gramaticales a través de una propuesta de carácter decididamente práctico, organizada en torno a ejercicios, cuestionarios y frecuentes prácticas de escritura. Se trata de un registro temprano del tipo de gramática escolar que, como se observó (Toscano y García, 2012) en relación con la de Henríquez Ureña y Binayán (1927), encuentra en la innovación de los procedimientos de enseñanza, y en particular en el énfasis en la práctica, una respuesta a lo que percibe como una ruptura del orden lingüístico producida por el proceso inmigratorio contemporáneo.

De esta forma, la obra de Pérez de Bertelli puede ser leída como un exponente de la renovación didáctica que impulsó el normalismo en la Argentina durante las primeras décadas del siglo xx. No obstante, su particular adopción del método intuitivo para la enseñanza de la gramática, que contaba para entonces con algunos antecedentes destacados, como los de Ferreyra (1898) y Figuera (1904), puso de manifiesto el interés de la autora por incorporar a la gramática escolar la reflexión sobre el método en enseñanza de lengua materna y por desplazar el exceso de teoría. La proposición de este cometido es enunciada programáticamente, tanto en el prólogo como en un artículo monográfico publicado el mismo año que esta obra, y asume la necesidad de recuperar el aspecto «científico» del conocimiento lingüístico como herramienta para favorecer la enseñanza de la lengua. Lo hace a partir de dos operaciones novedosas en la tradición escolar: la incorporación de la reflexión metateórica, y la construcción de un destinatario activo en la consideración sobre su propia variedad lingüística.

\section{Rita Pérez de Bertelli y la pedagogía de Amadeo Jacques}

Rita Pérez de Bertelli nació en Buenos Aires el 30 de mayo de 1880 y murió el 20 de diciembre de 1952.

3. Los datos sobre el corpus gramatical han sido recabados por los miembros del proyecto y, si bien han sido revelados parcialmente en Toscano y García, Lidgett y García Folgado (2019), su presentación cabal todavía permanece inédita.

4. La participación de la mujer en la enseñanza, sobre todo en la escuela primaria, se incrementa notablemente como consecuencia de la consolidación de la creación de las escuelas normales a partir de 1870. Como señaló Morgade (1993), entre 1874 y 1921, años de emergencia y consolidación del normalismo, se graduaron en la Argentina 2626 maestras y solo 504 maestros. Tanto las conferencias pedagógicas, organizadas tras la sanción de la ley de Educación Común de 1884, como las revistas vinculadas al ámbito escolar — sobre todo el Monitor - revelan también una considerable participación de las maestras en la discusión pedagógica. No obstante, como advirtió también Morgade (1993, p. 55) «los escasísimos documentos pertenecientes a mujeres maestras de fines de siglo y las primeras décadas del actual nos permiten inferir que su participación fundamental en el sistema educativo se produjo en un lugar de ejecución». 
Hacia la ciencia del lenguaje. Ejercicios de análisis gramatical (1916), de Rita Pérez de Bertelli

Luego de concluir sus estudios primarios y especiales se trasladó a la provincia de Tucumán, donde desarrolló una larga y sostenida actividad educativa. En 1915, es designada a cargo de la recientemente fundada cátedra de Puericultura, dependiente de la Escuela Pedagógica Sarmiento. Como señaló Barbieri (2017, p. 177), esta Escuela había sido incorporada en 1914 a la Universidad de Tucumán «bajo la dirección de la educadora normalista Otilde B. Toro, y constituyó un espacio abierto a concepciones pedagógicas como las de Ernesto Nelson, que ideó un plan para reformar la escuela secundaria sobre la base del activismo». A partir de 1918, la Escuela funcionó como institución incorporada a la Escuela Normal, hasta que, en 1922,

[...] se aprobaron los planes de estudios de «Visitadoras de Higiene» en la Escuela Sarmiento, de dos años de duración, que incluían cuatro asignaturas cada uno, relativas a nociones de anatomía y fisiología, deberes de las enfermeras, enfermedades sociales y de la primera infancia, primeros auxilios, etc. En este plano se destacó la labor de la Profesora Rita P. de Bertelli [...] (Barbieri 2017, p. 178).

La actividad de Pérez de Bertelli al frente de la cátedra de puericultura obtiene cierto reconocimiento en la prensa educativa de la época. Así, El Monitor de la Educación Común (1915) recoge una «Clase de puericultura en 3er. año», en la que la autora aspiraba a «familiarizar a las familias con la estadística, fuente de tantas y tan útiles sugestiones» (p. 503) a partir del lugar que ocupaba en «la cátedra de puericultura que me cupo la honra de iniciar, por mandato del actual gobernante, Dn. Ernesto Padilla» (p. 506).

Es en el marco de esta Universidad de Tucumán — Nacional de Tucumán a partir de 1921— donde desarrolló Pérez de Bertelli en gran medida su labor educativa. Una vez allí, se integró a la Liga Sanitaria del Norte, un ámbito de la extensión universitaria desde el que realizó una de sus publicaciones más importantes: Por la salud del niño: guía práctica de puericultura (1924), la cual «mereció el honor de un prólogo entusiasta del doctor Gregorio Aráoz Alfaro» (La Gaceta de Tucumán, 1 de abril de 1968, s.p.) ${ }^{5}$. Al respecto, de acuerdo con Barbieri (2017): «Esta guía para el cuidado de la salud se sumaba a los deberes de la maestra, que no sólo debía transmitir conocimientos sino que estaba obligada a prevenir enfermedades y educar en atención a ello» (p. 178).

La crítica ha señalado el vínculo de Pérez de Bertelli con algunos de los intelectuales de actuación más destacada en la universidad tucumana, lo que se conoce como Generación del Centenario: Ricardo Jaimes Freyre y, en particular, Juan B. Terán, fundador de la Universidad. Para Tacconi de Gómez, por ejemplo, corresponde a Terán «la edición de obras de mujeres a quienes indujo a publicar: doña Tránsito Cañete de Rivas Jordán y doña Rita P. de Bertelli» (2006, p. 369). No obstante, si en el recorrido biográfico de Pérez de Bertelli se nota un acercamiento a la Generación del Centenario, sus concepciones pedagógicas se pueden vincular directa y explícitamente con las propuestas de Amadeo Jacques, al que la autora, como una colaboradora habitual en la prensa tucumana de la época ${ }^{6}$, le dedicó en 1916, el mismo año en que publica Hacia la ciencia del lenguaje, un

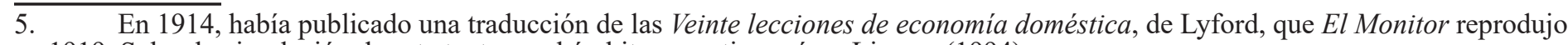
en 1919. Sobre la circulación de este texto en el ámbito argentino, véase Liernur (1994).

6. Según La Gaceta de Tucumán (1968, s.p.), «las columnas de La Gaceta, El Orden, De Norte, entre otros, la tuvieron como colaboradora asidua». Hemos podido localizar, sin embargo, pocas de estas colaboraciones, en general de corte patriótico: Pérez de Bertelli (1916b) es un elogio del espíritu independentista escrito con motivo del centenario de la independencia; también lo es Pérez de Bertelli (1938), que recoge un discurso pronunciado por la autora en ocasión del «centésimo décimo sexto aniversario» del «glorioso advenimiento» de la patria. Agradecimientos a César Canceco (Biblioteca Leoni Pinto ISES-CONICET/Tucumán) por el acceso a esta documentación. 
Hacia la ciencia del lenguaje. Ejercicios de análisis gramatical (1916), de Rita Pérez de Bertelli extenso trabajo: «Acción educativa de Amadeo Jacques en Tucumán». Se trata de un texto significativo porque, en la medida en que allí Pérez de Bertelli empatizó y valoró positivamente la acción educativa de Jacques, la autora manifestó sentirse identificada con las ideas pedagógicas de este último.

El extenso artículo se publicó en el Álbum general de la provincia de Tucumán en el primer centenario de la independencia argentina, en el cual se busca reconstruir e interpretar la actuación de Amadeo Jacques (18131865), con especial atención a su actividad en Tucumán. El trabajo comienza con la traducción que Pérez de Bertelli ofrece de una carta de Jacques a Paul Groussac del 10 de enero de 1853. Allí Jacques, desde Montevideo, se presenta y le pide a Groussac que intervenga ante Justo José de Urquiza para conseguirle trabajo. En esa presentación, dice:

Mi propósito era, y es aún, ejercer mi antigua profesión. Solamente he creído deber al cambiar de región mudar también de materias de enseñanza: de profesor de Filosofía que era me he convertido de Física, de Química y de Mecánica.

Me parece que en un país tan joven sería inútil y casi ridículo traer especulaciones metafísicas y que lo que conviene más es ante todo una enseñanza práctica de las ciencias que tienen su aplicación directa, ya a la agricultura, ya a la industria (Pérez de Bertelli, 1916a, s.p.).

Para Jacques, América supone una suerte de reconversión epistemológica; la idea de una «enseñanza práctica de las ciencias» va asociada a otra idea, la de que la especulación teórica no se justifica en una joven nación:

Sabéis, poco más o menos, por lo que os he dicho de mí, para lo que puedo ser apto. Es, por lo pronto, para instalar bajo una u otra forma, una enseñanza científica poco elevada pero muy práctica y cuyo objeto principal sería no formar sabios; sino hombres útiles e ingenieros prácticos, contramaestres y jefes para las explotaciones agrícolas, nacidas o por nacer (Pérez de Bertelli, 1916a, s.p.).

Ese énfasis en la práctica en desmedro de la enseñanza teórica, que articula el pensamiento de Jacques, es una de las ideas centrales del texto gramatical que Pérez de Bertelli escribió este mismo año, como se podrá notar más adelante.

Según el relato de Pérez de Bertelli, las gestiones llevadas a cabo por Groussac son exitosas, aunque tardíamente; así, en agosto de 1855 Urquiza comisionó a Jacques para realizar una misión en Tucumán destinada a «a estudiar la geografía de dicha provincia, a reconocer sus minas y a procurarse los conocimientos cosmográficos que sean necesarios para completar su trabajo» (Pérez de Bertelli 1916a, s.p.): un tipo de acción antes «práctica» que educativa o «científica» en los términos de Jacques. La incorporación de este al campo educativo tucumano, sin embargo, se produjo pocos años después, cuando, el gobernador de Tucumán, Agustín Justo de la Vega ${ }^{7}$, lo designó como director del Colegio San Miguel tras su reapertura en abril de 1858, siendo fundada en octubre de 1857 como la primera escuela secundaria de Tucumán. El plan de estudios que para ese Colegio presentó el pedagogo francés al gobernador de la Vega plasma su concepción anterior; en relación con la enseñanza de la lengua, estableció un criterio esencialmente comunicativo: «Idioma gramatical. Ortografía y Literatura Castellana; ejercicios de dicción oral y de redacción de cartas, notas, memorias, discursos, relaciones, descripciones, etc.»

7. Cargo que conservó hasta el $1^{\circ}$ de septiembre de 1872, cuando renunció a su cargo como director del Colegio San Miguel. 
(Pérez de Bertelli, 1916a, s.p.).

Unos días después, Jacques publicó en El Eco del Norte una suerte de justificación general de su plan de estudios, asentada sobre las mismas ideas de saber útil y práctico:

Ese es efectivamente mi pensamiento dominante, en el sentido en que debe ser dirigida la juventud argentina; es el de las ciencias útiles, de aquellas ciencias que tienen en la vida material, en las profesiones mercantiles, en la industria agrícola y pastoril, y en cuanto se refiere al desarrollo y engrandecimiento de la riqueza pública y privada, aplicaciones de cada día y de cada momento. [...]

No aspiramos a formar sabios, pero sí hombres útiles y prácticos, que sepan en cualquier circunstancia que les tenga reservada el porvenir evitar la pobreza; recurriendo al arte para aprovechar la riqueza natural de su suelo natal (1916a, s.p.).

La justificación le permite, luego, un largo alegato contra las humanidades, que representarían el tipo de saber especulativo e innecesario que domina para él el contexto educativo actual y debe ser combatido:

[...] es preciso considerar que las letras, especialmente latinas o de antigüedad, son un lujo.

Primero, pues, que el lujo se debe atender a lo necesario. En la Confederación Argentina sobran los doctores (perdónenme tamaña herejía).

La tendencia casi exclusivamente literaria y filosófica de la Universidad de Córdoba proporciona solamente una instrucción como para entretener y adornar el espíritu.

De ahí dimana un mal grande, y es la aspiración demasiado general a los oficios administrativos y empleos políticos (Pérez de Bertelli, 1916a, s.p. $)^{8}$.

Como se indicó anteriormente, la autora no solo se limitó a reconstruir la tarea de Jacques en Tucumán, sino que también la valoró, empatizó con ella y, todavía más, vio en él la figura de un iniciador de la dinámica educativa universitaria de la que ella misma participó y que se caracterizó por la fidelidad que mantiene respecto del ideario del pedagogo francés:

[...] este hombre superior que se adelantaba con paso firme cincuenta años a su tiempo, como bien se advierte al través de sus proyectos, trazando derroteros que son todavía nuevos, y que la fundación de la Universidad de Tucumán y el Gobierno del doctor Ernesto Padilla han setualizado [sic] entre nosotros (Pérez de Bertelli 1916a, p. $\mathrm{x})$.

Ese ideario se verifica, como se verá, en Hacia la ciencia del lenguaje. Ejercicios de análisis gramatical (1916), trabajo que fue el resultado y la compañía de su actividad docente, esta vez en «las cátedras de Castellano y de Literatura tanto en la antigua Escuela Superior Nacional de Comercio, de la que egresaron las primeras camadas tucumanas de contadores y en la Escuela y Liceo Vocacional Sarmiento» (La Gaceta de Tucumán, 18 de octubre de 1982, s.p.).

8. Con relación a la enseñanza del latín Jacques afirmó: «las reglas de la gramática, abstractas y vacías cuando van solas» (Pérez de Bertelli 1916, s.p.). 


\section{Una gramática para la formación de maestros}

\subsection{Contra la monotonía de los gramáticos}

Como algunas otras obras del corpus, la de Pérez de Bertelli no es una gramática elaborada para los niños en edad escolar, sino para los maestros. Este tipo de obras, cuyo primer antecedente en el corpus argentino es el de Martí (1876) $)^{9}$, comenzaron a circular como consecuencia de la creación de las escuelas normales, dedicadas a la formación de maestros, luego de la década de 1870. Por tratarse de obras destinadas a los docentes más que a los alumnos, algunas de estas gramáticas exhiben un abordaje teórico más elaborado de los contenidos, mientras que otras, como la que es objeto de este artículo, dedican un mayor espacio a la presentación de modelos de ejercicios para ser utilizados en el aula. Lo característico de todas ellas, no obstante, es su preocupación por los métodos pedagógicos; una preocupación que el normalismo logró instalar en el ámbito escolar argentino desde sus orígenes.

En línea con esta corriente normalista todavía emergente a fines del siglo XIX, Pérez de Bertelli incluyó en su gramática un prólogo en el que se ocupó de criticar el método tradicional de enseñanza de la lengua. Desplegó en el mismo, como en muchas otras gramáticas, una declaración de los principios que motivaron la escritura de la obra:

La rutina a que ha obedecido y sigue obedeciendo la enseñanza de la gramática así como la esterilidad de su estudio [...] me decidió a idear para mis clases un procedimiento que despojara a las lecciones de la infructuosa monotonía, y me alejara del desorden en que se cae cuando en el deseo de reaccionar contra las inútiles definiciones repetidas en virtud de la memoria de frases, se entra de lleno en el análisis sin base alguna por parte del alumno (1916c, p. 3).

Si por un lado se trata de alejarse de la tan recurrente monotonía de las reglas a la que tradicionalmente se plegaba la enseñanza de la gramática, por el otro resulta indispensable para la autora ofrecer un procedimiento ordenado que permitiera construir la enseñanza teórica sobre una base sólida. En ese sentido, no hay en la propuesta de esta autora una oposición, frecuente en el discurso normalista, entre el método y la teoría, sino la necesidad de armonizar ambos aspectos para alcanzar el objetivo último de que el alumno interiorizara los conceptos teóricos. Pérez de Bertelli retomó en este aspecto la tradición inaugurada por Jacques, y así lo hizo saber también en el prólogo. cuando se refirió a los consejos de su «sabio maestro»:

La teoría ennoblece la práctica», decía Amadeo Jacques [...]; y el estudio del idioma no puede constituir excepción.

El secreto consiste en hacer fácilmente asequible la teoría, e «imitando a la naturaleza, untar con miel los bordes de la copa para hacer tragar el brebaje de la ciencia (1916c, 5).

Con la ciencia como objetivo último, según lo anuncia el mismo título de la obra, Hacia la ciencia del lenguaje, Pérez de Bertelli diseñó su método de enseñanza sobre la base de dos operaciones que considera fundamentales, tanto en la ciencia como en la enseñanza: la observación y la reflexión. Para la autora, «todo se reduce a que el niño observe atenta e inteligentemente las frases de cada ejercicio para llegar por el estudio de

9. $\quad$ Para un estudio detallado de la obra de este autor, véase Toscano y García y García Folgado (2015). 
Hacia la ciencia del lenguaje. Ejercicios de análisis gramatical (1916), de Rita Pérez de Bertelli los casos particulares que encauzan el análisis, a deducir y generalizar el uso de las palabras» (1916c, p. 8). Pero esa observación requiere que el alumno implique su atención en la enseñanza. Y para captarla, Pérez de Bertelli consideraba fundamental trabajar con lo que denominaba «lenguaje familiar», es decir, un lenguaje que, en lugar de imitar a la lengua literaria - tan ponderada en otras gramáticas escolares basadas en los modelos positivos que debían imitarse - , resulte más cercano a la lengua hablada y escuchada por los niños en sus hogares:

Bien se ve que he dado preferencia al lenguaje familiar y a las causas diarias que lo provocan [...]. He querido con ello que el alumno deseche la idea de que la posesión de las reglas gramaticales es privilegio de doctos y de sabios; que se convenza, antes bien, de que ellas están al alcance de todas las formas; desde las más modestas hasta las más ricas; desde las más llanas hasta las más aladas y sutiles (1916c, p. 8).

Este punto es fundamental, porque si la mayor parte de las gramáticas del corpus pueden ser confrontadas en función del nivel metalingüístico, es decir, de las opciones terminológicas y conceptuales elegidas para explicar la lengua, en este caso, la oposición a las gramáticas precedentes también alcanza el nivel del lenguaje objeto ${ }^{10}$ : ya no se trata de describir la lengua literaria, la lengua de los hombres doctos, sino una lengua familiar, que, como la anterior, es una construcción de la gramática, pero que intenta imitar un modelo distinto. Esa operación, aunque artificiosa, es en sí misma un cambio trascendental dentro de los modelos de enseñanza de la gramática. Precisamente porque, al desplazar el modelo de la lengua objeto, desplaza también el criterio de lo que se define como correcto. De allí que la definición de la norma en la gramática de Pérez de Bertelli, como se verá, resulta más compleja que la propuesta por la mayor parte de las obras del corpus gramatical escolar.

Tal elección en el plano de la lengua objeto impulsó también una necesaria revisión a nivel del metalenguaje. La explicación de los fenómenos gramaticales registrados en ese lenguaje familiar que se pretende describir invoca la necesidad de cuestionar preceptos teóricos y de adaptar la teoría al uso. En este punto, la gramática exhibe la reflexión metalingüística como una de sus características más importantes:

El eclecticismo ha guiado la elección de los ejercicios de análisis tratando de colmar con las tesis de unos autores las lagunas que otros dejan; y desechando de éstos y de aquéllos teorías demasiado complejas para ser penetradas en sexto grado o en primer año normal, y a las que llegarán por sí solos, fuera del aula, los alumnos que recogieren en este libro la simiente de la investigación arrojada en el transcurso de sus líneas (1916c, p. 8).

De esta forma, asumiendo uno de los postulados centrales del normalismo, Pérez de Bertelli decidió adaptar las teorías a la práctica docente, proponiendo descartar el exceso de complejidad teórica a favor de una propuesta gradual del aprendizaje. La adaptación de la teoría planteada tanto a la descripción del lenguaje cotidiano que la autora construyó como objeto de su gramática como a la gradualidad de la práctica docente, requería de cierto eclecticismo a la hora de incorporar las categorías teóricas. Esta tarea, si bien desliga la obra de toda pretensión doctrinaria, no le impide desplegar en sus páginas la reflexión metalingüística necesaria para cuestionar la imprecisión de algunos conceptos, contrastando las caracterizaciones de prestigiosos gramáticos con

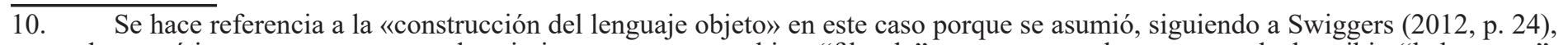
que toda gramática, en tanto que texto descriptivo, presenta «un objeto "filtrado" con respecto a lo que pretende describir: "la lengua x"; de este último objeto "material", una gramática siempre nos brinda una "imagen" selectiva, parcial». 
Hacia la ciencia del lenguaje. Ejercicios de análisis gramatical (1916), de Rita Pérez de Bertelli

los fenómenos observados por los alumnos en los ejemplos (véase 3.2.). El objetivo central del asunto, tal y como lo señaló la autora, no consiste en «hacer una gramática» — «empresa inútil de suyo después de los monumentos que los grandes preceptistas han levantado»— sino lograr que todos reciban «el impulso que los encamine hacia la ciencia del lenguaje» (1916c, p. 16).

\subsection{El método de Pérez de Bertelli}

La gramática de Pérez de Bertelli, como ya se mencionó, es una obra pensada para la formación de docentes. Sus destinatarios son los educadores en formación y no los niños en edad escolar, pues, según afirmó la autora, «si los libros de texto son imprescindibles para consulta por parte del maestro, son inútiles para los alumnos, porque carecen de valor sugerente»; ellos conducen, por el contrario, a que el niño acepte «reglas y preceptos hechos repitiéndolos sólo para retenerlos textualmente en el momento de la clase» (1916c, p. 7). Sin embargo, toda la obra está presentada como si reprodujera la práctica docente en el aula, que sí está destinada al niño. Esto produce, por lo tanto, un efecto de duplicidad en el destinatario: mientras que el libro se destina expresamente al docente y funciona como una herramienta para la organización de la clase, el contenido de los ejercicios y cuestionarios que presenta asume directamente al estudiante escolar como su destinatario. Para lograr este efecto, la gramática se organiza en 10 series de 30 ejercicios, seguidos cada uno de 30 cuestionarios; una «serie última», destinada a proponer una clasificación funcional de las clases de palabras; y una «Revisión del análisis gramatical». Como se muestra en la Tabla 1, todas las series, con la excepción de la octava y de la «Revisión», se dedican a los contenidos de analogía, pues, como lo explicó la autora en el prólogo de la obra, «quien sabe analogía tiene la puerta abierta para penetrar en las obscuras encrucijadas de la sintaxis», mientras que «los secretos de las funciones lógicas de las palabras serán, en cambio, impenetrables para el que no haya aguzado su espíritu en la observación de las funciones gramaticales» (1916c, pp. 15-16). 


\begin{tabular}{|c|c|}
\hline Serie & Contenido \\
\hline Serie 1 & Sustantivos \\
\hline Serie 2 & Artículos \\
\hline Serie 3 & Adjetivos y determinativos \\
\hline Serie 4 & Pronombres \\
\hline Serie 5 & Verbos \\
\hline Serie 6 & Adverbios \\
\hline Serie 7 & Complementos del verbo \\
\hline Serie 8 & Conjunciones \\
\hline Serie 9 & Interjecciones \\
\hline Serie 10 & \\
\hline Serie última & Redefinición de las clases de palabras y sus funciones \\
\hline Revisión de análisis gramatical & \\
\hline
\end{tabular}

Tabla 1. Macroestructura de la obra

La preeminencia de la analogía sobre la sintaxis no solo responde a la asignación curricular propia de la escuela primaria, sino también al método intuitivo que la autora propuso. En efecto, como se vio tanto en el prólogo de la gramática como en su artículo sobre Jacques, el objetivo de la enseñanza para Pérez de Bertelli consiste en imitar el procedimiento de observación y experimentación propio de las ciencias; es decir, buscar avanzar desde los elementos más simples a los más complejos en un proceso de síntesis, que es opuesto, precisamente, al método analítico dominante en la tradición gramatical previa al normalismo.

Así, por ejemplo, en el Ejercicio 1 que inicia la primera serie, se parte de un texto en el que, a partir de la consiga propuesta, se espera que los alumnos encuentren los tipos de palabras que se ajustan a las definiciones posibles del sustantivo: «Anote las palabras que nombran SERES (personas, animales, vegetales minerales), OBJETOS, CUALIDADES en abstracto [...]» (los cambios de tipografía aparecen en el texto original). Una vez cumplida esta consigna, se procede al primer cuestionario: «De todas las palabras que Vd. ha anotado ¿cuáles se aplican a un solo ser, a una sola ciudad para distinguirlos de los demás de su misma esencia o especie...?» (1916c, pp. 17-18). En líneas generales, el procedimiento consiste en partir de un uso específico del lenguaje (en este caso, un texto descriptivo de la naturaleza), un texto o una situación comunicativa determinada, y desde allí proponer una serie de ejercicios en los que se conduce al alumno a reconocer las partes del discurso sin introducir todavía los conceptos específicos. A continuación, se introduce el cuestionario, cuyo objetivo consiste en que el alumno trate de llegar por inducción al reconocimiento de las categorías gramaticales y de sus clasificaciones. A medida que se avanza en el cuestionario, comienzan a introducirse algunos conceptos: «¿con qué letra inicial se escriben los substantivos propios? [...] ¿Diga cuántos substantivos comunes y cuántos propios ha encontrado al observar el trozo del ejercicio I?». Tras complejizar progresivamente las interrogaciones, se pide que el alumno 
Hacia la ciencia del lenguaje. Ejercicios de análisis gramatical (1916), de Rita Pérez de Bertelli ensaye alguna definición, no sin antes indicar las posibles dificultades de semejante tarea: «por los numerosos conceptos que el substantivo es capaz de expresar, por la multiplicidad de sus aspectos, ¿cree Vd. fácil dar una definición de substantivo? Ensaye algunas...» (1916c, p. 19). La gramática procede así, gradualmente, desde los conceptos más simples hasta sus clasificaciones más específicas, avanzando en pares de ejercicios y cuestionarios, destinados aquellos a la observación y al reconocimiento intuitivo, y estos a la introducción de los conceptos teóricos.

Las cuatro características esgrimidas hasta este punto - a saber, la duplicidad del receptor (docentes y alumnos), la asunción del método intuitivo, la presentación gradual de los contenidos y la preeminencia de la ejercitación — no son ajenas a la tradición normalista que se consolidó durante las primeras décadas del siglo xx. Otras gramáticas del corpus, como las de Ferreyra (1898) y Figueira (1904), apelan a este tipo de recursos con fines similares a los que esgrimió Pérez de Bertelli en su prólogo. En cambio, hay otros dos recursos que utiliza esta autora que verdaderamente constituyeron una novedad para la gramática escolar: la introducción de la reflexión metalingüística en la práctica y la construcción de un lenguaje objeto altamente receptivo de los usos lingüísticos.

Por reflexión metalingüística se entiende, en este caso, la evaluación de definiciones y categorizaciones propuestas por autoridades en materia gramatical, o por la misma tradición escolar. Es un procedimiento no muy frecuente en las gramáticas escolares y que, normalmente, cuando se registra, suele estar relegado a notas marginales o a explicaciones destinadas al docente. ${ }^{11}$ Lo curioso del caso que ocupa la atención de este artículo es precisamente que la misma ejercitación propuesta para el alumno escolar incluye de manera solapada muchas veces una reflexión crítica sobre determinados conceptos. Así, por ejemplo, el cuestionario antes referido sobre el sustantivo, tras conducir al alumno hacia el reconocimiento de las subclases de sustantivos, agrega la siguiente consigna:

Observe si los substantivos que anotó aumentan la comprensión de otra u otras palabras agregándoles otras ideas; es decir, observe si son determinantes.

Aplicando el fruto de esta observación, opine sobre la siguiente manera de definir el substantivo: «es toda aquella palabra que no expresa la finalidad de la elocución y que no es determinante de otra u otras palabras».

¿Resiste a toda objeción? ¿No involucra también a las palabras que reemplazan al nombre y a las que expresan la acción en abstracto (tú eres bueno, correr es saludable) [...]? Modifique la definición de referencia (1916c, p. 20).

La ejercitación incluye, de esta forma, la posibilidad de que los alumnos efectivamente participen de la reflexión sobre las categorías gramaticales, en este caso a partir de un cuestionamiento sobre una definición de sustantivo, atribuida por la misma autora a Benot.

Este recurso se reitera en otras partes del texto y alcanza su mayor eficacia en la última serie, dedicada a la redefinición de las clases de palabras. Resulta pertinente detenerse en esta sección de la obra porque es en tal

11. Un caso de este tipo es analizado para la gramática de Atienza y Medrano (1896) en Lidgett (2014) y para la gramática de Bastianini en Toscano y García y García Folgado (2017). Se trata en estos casos de gramáticas para la escuela secundaria, en las que la reflexión metateórica se destaca en diferentes tipografías o en notas específicas. 
Hacia la ciencia del lenguaje. Ejercicios de análisis gramatical (1916), de Rita Pérez de Bertelli punto donde la autora sintetizó su propuesta para el estudio de la analogía. Consecuente con el método intuitivo que puso en práctica, el procedimiento que Pérez de Bertelli utilizó para lograr esta síntesis no consistía en la exposición lisa y llana de su propuesta teórica, sino en una ejercitación en la que el alumno debía comparar una serie de categorizaciones y reflexionar sobre su aceptabilidad. Así, el primer ejercicio de esta serie solicita al alumno que «medite» sobre algunas teorías mencionadas a lo largo del libro. Enumera, a continuación, tres de ellas: la teoría benotiana que propone una clasificación funcional de las clases de palabra —a partir de la incorporación de las nociones de sustantivos frase, sustantivos oración, adjetivos frase, adjetivos oración, etc.-—; la propuesta que «solo considera siete partes de la oración, porque suprime el artículo y el pronombre; desde que asimila el artículo y el participio al adjetivo; y el pronombre al verbo»; y la que, inspirada en la gramática filosófica, «reduce a tres clases todas las palabras: substantivas (nombre y pronombre), modificativas (adjetivos, modificativos y adverbios) y conexivas (verbos, preposiciones y conjunciones)» (1916c, p. 257). A continuación, el ejercicio propone las siguientes consignas:

Después del estudio que ha hecho Vd. de las palabras para deducir el oficio gramatical que desempeña cada una, ¿sigue creyendo que hay partes de la oración, o piensa que la oración tiene partes, las cuales desempeñan diferentes oficios gramaticales según las relaciones que tienen entre sí?

Cuando Vd. vea una palabra aislada ¿podrá decir qué oficio gramatical desempeña? Canto, corro, era, pienso, son, ser, entre muchísimas otras, ¿son verbos?, ¿son substantivos? ¿No es menester relacionarlas con otras palabras en la oración, para responder?

Luego, ¿«analogía es la parte de la gramática que enseña el valor de las palabras consideradas aisladamente y según sus varios oficios» o la analogía enseña a conocer el oficio gramatical que las palabras desempeñan en la oración? (1916c, p. 257).

La sucesión de interrogantes, evidentemente, conduce al alumno hacia una respuesta determinada que coincide con la propuesta de la autora de que la única clasificación posible es la funcional, pero lo hace incitando a que sea el propio alumno el que llegue a esta conclusión, imitando la labor del teórico. De esta forma, Pérez de Bertelli buscó poner en práctica su peculiar propuesta de enseñanza, en la que asumía que el aprendizaje se construye siguiendo el mismo modelo con el que se construye el conocimiento científico. La inclusión de la reflexión metalingüística entre las prácticas escolares, de donde normalmente se excluye, parece así justificarse en razón de este objetivo.

El segundo de los procedimientos disruptivos que ya se habían mencionado anteriormente es el de la construcción de un lenguaje objeto receptivo de las variedades lingüísticas. Procedimiento que, como se pudo apreciar, es anunciado también en el prólogo, cuando se enfatiza en la necesidad de trabajar con el «lenguaje familiar» (véase 3.2). Esto no supone, necesariamente la suspensión de todo juicio prescriptivo, sino la incorporación en el texto de ejemplos extraídos del lenguaje cotidiano y que intentan, por lo tanto, registrar de algún modo los fenómenos de variación.

Un ejemplo de la implementación de este recurso se puede observar en uno de los ejercicios de la serie segunda, que trata sobre el artículo. En el mismo, se incluyen varias listas de enunciados y se pide al alumno que «recuerde a quienes oyó emplear así o semejantemente el artículo»: 
a) La Lola me dijo que vendría.

La Luisa es encantadora.

El pedro te entregará la carta.

b) La Duse fue llamada «la divina».

La Barrientos tiene una garganta privilegiada.

La Patti recorrió gloriosamente los escenarios del mundo.

c) Parece que las Morias nos hubiéramos dado cita en esta reunión.

Esta es la Familia de los Pedros (Pérez de Bertelli, 1916c, p. 67).

Siguiendo el método exhibido hasta ahora, la autora también incluyó un cuestionario relacionado con las oraciones anteriores, en el que se espera que el alumno reflexione a partir de las preguntas: «¿Oyó Vd. decir entre nosotros, en lenguaje culto, la Lola, la Luisa, el Pedro?»; «iImitaría Vd. tales construcciones?»; «En cambio ¿recuerda Vd. haber oído o leído en lenguaje culto, frases como los ejemplos del grupo b?»; «¿Qué deduce Vd. de ello, respecto al uso del artículo cuando precede a nombres de mujeres?»; «¿Qué le permite opinar su observación sobre la corrección del uso del artículo delante de los nombres propios pluralizados, como en los ejemplos de $c$ ?» (Pérez de Bertelli, 1916c, p. 67). Como es evidente, estos interrogantes conducen al alumno a contrastar entre los usos aceptados por el denominado «lenguaje culto», como serían los de las series b y c, y los usos no aceptados por esta variedad, como los de la serie a. En esto reside, precisamente una de las particularidades de esta gramática; porque, si bien la ejercitación a través de modelos negativos — es decir, de ejemplos de usos incorrectos que deben ser corregidos por el alumno- es, de hecho, un recurso bastante extendido entre las gramáticas de comienzos del siglo xx (Di Tullio, 2002; López García, 2009), mas no lo es del mismo modo el registro de la variación ${ }^{12}$.

La presencia de la variación lingüística en esta gramática, por otro lado, no se reduce al contraste entre los usos «vulgares» y los «cultos». Pérez de Bertelli también registró la distancia entre la norma culta peninsular, que funcionaba como el criterio de corrección para la mayoría de las gramáticas escolares argentinas del momento, y la norma culta regional. Así, por ejemplo, en la revisión del análisis gramatical, a la que dedica la última sección del libro, la autora ejemplificó el análisis con las siguientes palabras, atribuidas al poeta Ricardo Rojas:

Ese día, el peón doméstico y yo, niño aún, cruzábamos a caballo una comarca triste, en tierra santiagueña. Dos horas hay en la región propicias al misterio satánico: la siesta con la desolación de sus senderos, la noche con sus sombras (1916c, p. 280).

Al finalizar el análisis, agregó una nota en la que destacó que, así como la palabra «santiagueña» no se encuentra en el diccionario de la Real Academia Española en su acepción utilizada en el ejemplo, «se emplean en el libro algunas más que no figuran en el léxico oficial» pero que sí han sido «autorizadas por el uso de buenos escritores» (1916c, p 280). A continuación, agregó:

12. La gramática de Pérez de Bertelli sigue en este aspecto una línea que integran otras obras del corpus, también atentas al registro de la variación. Entre ellas, cabe mencionar los casos de Sastre (1857), Atienza y Medrano (1896) y Vera y González (1905), que analizó Arnoux (2017); y los ya mencionados de Martí (1876) y Henríquez Ureña y Binayán (1927). 
La índole ecléctica del presente trabajo, destinado a sugerir ideas, no podía imponer el establecimiento del idioma. Antes bien, el maestro que haya sacado provecho de sus lecciones, verá con íntima satisfacción cómo sus discípulos se dividen en leístas y loístas, por ejemplo: cómo éstos respetan a la Academia Española empleando a les en dativo únicamente, y cómo aquéllos encuentran muy grato su uso, ya divulgado, en acusativo; [...] cómo determinado bando se decide por el castellano del siglo de oro, mientras tal otro prefiere el castellano de oro del siglo, en manos de orfebres americanos cual Rubén Darío, Arturo Capdevilla, Ricardo Rojas, etc., predilección que se revelará en los ejemplos que el alumno haya buscado o creado; cómo, en fin, a la apatía por un estudio, han sucedido el interés y el gusto manifiestos (1916c, p. 281).

La gramática de Pérez de Bertelli confirma, de este modo, su elección por no restringir el criterio de corrección exclusivamente a la norma culta peninsular. Se trata de una posición singular en un contexto en el que la adopción de esta norma constituía la elección más frecuente para una gramática escolar que había encontrado en los instrumentos lingüísticos de la Real Academia Española un sustento confiable para la prescripción normativa y la uniformidad doctrinaria. En ese sentido, como sostuvo López García (2009), la falta de reflexión sobre la variedad lingüística local durante las primeras décadas del siglo xx produjo «una contradicción entre el uso en la enseñanza de una norma ideal, alejada de la norma (culta) de la región y la aplicación de estrategias metodológicas vinculadas con perspectivas comunicativas ligadas a prácticas de enseñanza eminentemente orales» (p. 11). Frente a este panorama, la posición de Pérez de Bertelli en su gramática puede leerse como un intento de resolver aquel dilema a partir del cuestionamiento de la uniformidad del concepto de norma culta.

\section{Conclusión}

La gramática de Pérez de Bertelli asume un lugar destacado dentro del corpus de la gramática escolar argentina, no solo por tratarse de una de las pocas obras escritas por una mujer en un contexto que evidentemente no favorecía la participación de mujeres en el mercado editorial, sino también por la originalidad de su propuesta metodológica, que pretendía dar respuesta a los inconvenientes que la expansión del sistema educativo había puesto de manifiesto en relación con la enseñanza de la lengua materna.

En ese sentido, como se señaló inicialmente, durante las últimas décadas del siglo xix y las primeras de Xx, el sistema educativo atravesaba una crisis que dio lugar a una serie de cuestionamientos en torno a los objetivos y a la metodología de la enseñanza de la gramática. La primera respuesta a esta crisis consistió en la búsqueda e incorporación de nuevos contenidos teóricos que rivalizaron con los paradigmas consolidados para la enseñanza de la gramática — en particular, el de la Real Academia Española_ — La incorporación en el corpus escolar de la propuesta teórica de Andrés Bello y de la lingüística histórica y comparada durante la segunda mitad del siglo XIX, y la de Eduardo Benot durante las primeras décadas del xx constituyeron, sin lugar a dudas, claros ejemplos del intento de la gramática escolar por buscar una respuesta a los problemas derivados del carácter doctrinario y memorístico que se le endilgaba a su enseñanza en aquel entonces.

Otro tipo de respuesta fue la que ensayó el normalismo desde sus inicios en la década de 1870, sobre todo 
Hacia la ciencia del lenguaje. Ejercicios de análisis gramatical (1916), de Rita Pérez de Bertelli en el nivel primario y en la formación de maestros. A la renovación de los contenidos y de los paradigmas científicos, los normalistas buscaron sumar la necesidad de reformar los métodos de enseñanza. La preeminencia de la práctica sobre la teoría, la consideración de la gradualidad de los ejercicios, la adecuación de los contenidos a las capacidades de los alumnos y el acercamiento intuitivo a la teoría fueron algunas de las principales propuestas que se lograron implementar durante las primeras décadas del siglo xx para renovar los métodos didácticos.

La gramática de Pérez de Bertelli que fue analizada en este artículo se inscribe claramente en el impulso renovador que el normalismo pretendió otorgarle a la enseñanza. Como en otras gramáticas, también exponentes de las propuestas normalistas, la obra de Pérez de Bertelli cuestionó la enseñanza rutinaria y memorística de la gramática y opuso a ese modelo una propuesta basada en el método intuitivo, según el cual era el alumno el que debía alcanzar los conceptos desde su propia reflexión y a partir de una práctica dirigida por el docente. Sin embargo, a diferencia de otras propuestas similares, Pérez de Bertelli implementó en su texto dos procedimientos poco frecuentes en el corpus gramatical: la incorporación de la reflexión metalingüística y la definición de un lenguaje objeto que pone de manifiesto el fenómeno de la variación. El primero le permitió a la autora asumir una perspectiva crítica frente a ciertas definiciones o clasificaciones propuestas por las autoridades académicas, al tiempo que, en consonancia con el método propuesto, también le otorgó al alumno un espacio de reflexión teórica que contrasta con la enseñanza dogmática y preceptista. El segundo le posibilitó tomar distancia de otro aspecto característico del conjunto de textos gramaticales de la época: la uniformidad normativa. Al asumir en su trabajo la necesidad de usar ejemplos extraídos del lenguaje familiar y de la literatura local, la autora puso en evidencia el fenómeno de la variación y, al mismo tiempo, destacó la existencia de una norma culta distinta del español peninsular. Ambos procedimientos evidenciaron así una preocupación teórica y metodológica, que apunta no solo a la renovación didáctica, sino también a la teórica y la conceptual. 


\section{Referencias bibliográficas}

1. Arnoux, E. (2017). El tratamiento de la diferencia lingüística en las gramáticas para la escuela secundaria argentina de fines del siglo xix y comienzos del xx. Boletín de la Sociedad Española de Historiografía Lingüística, $11,31-53$.

2. Atienza y Medrano, A. (1896). Lecciones de idioma castellano. Buenos Aires: Félix Lojuane Editorial.

3. Barbieri, M. I. (2017). Reformas educativas, construcción y reconstrucción de ciudadanía en escuelas primarias y secundarias tucumanas. Continuidades y cambios entre 1915 y 1955. En B. E. Altabef(Coord.). La conformación del sistema educativo en Tucumán. Antecedentes, etapas y agentes. Consensos y resistencias (pp. 166-229). Tucumán: CFI.

4. Barcia, P. L. (2004). La primera autora argentina de una gramática. Boletín de la Academia Argentina de Letras, 69(271-272), 41-58.

5. Calero Vaquera, M. L. (2008). Una muestra de la presencia en Hispanoamérica del análisis lógico y gramatical: El Tratado (Buenos Aires, 1880) de V. García Aguilera. Ed.Uco, 3, 27-39.

6. Calero Vaquera, M. L. (2009). Apuntes sobre el Curso gradual de gramática castellana (ca. 1930) de José Hidalgo Martínez y su lugar en la tradición escolar argentina. Revista argentina de historiografía lingüistica, 1(2), 151-174.

7. Colangelo, M. A. (2012). La crianza en disputa: medicalización del cuidado infantil en la Argentina, entre 1890 y 1930. La Plata: Universidad Nacional de La Plata. (Tesis doctoral). Mimeo.

8. Di Tullio, Á. (2002). La otra crisis de la gramática escolar. En G. Parodi(Ed.), Lingüística e Interdisciplinariedad: Desafios del nuevo milenio. Ensayos en Honor a Marianne Peronard (pp. 281-295). Valparaíso: Ediciones Universitarias de la Universidad Católica de Valparaíso.

9. Ferreyra, A. (1894). Lecciones de idioma castellano. Arregladas a los programas oficiales del Colegio Nacional, escuelas normales y de comercio. Buenos Aires: Librería San Jorge.

10. Ferreyra, A. (1898). Curso completo de idioma nacional: gramática castellana. Obra adoptada como texto por el Consejo Nacional de Educación. Libro primero. Buenos Aires: Estrada.

11. Figueira, J. H. (1904). Un buen amigo. Libro tercero de lectura y ortografía. Buenos Aires: Cabaut y Cía. Editores.

12. García Folgado, M. J. (2010). Estudiar gramática bajo un orden analítico: la obra de Felipe Senillosa (1817).

En Encinas Manterola, M. T. et al. (Eds.). Ars longa. Diez años de AJIHLE (pp. 663-678). Buenos Aires: Ediciones Voces del Sur.

13. García Velloso, J. J. (1907). Gramática de la lengua castellana. Buenos Aires: Estrada.

14. Gaviño Rodríguez, V. (2017). La enseñanza del castellano en la Argentina de finales del xix y principios del xx. Textos recomendados y actitudes en torno a la lengua. Revista Argentina de Historiografía Lingüística, 9(1), 43-58. 
Hacia la ciencia del lenguaje. Ejercicios de análisis gramatical (1916), de Rita Pérez de Bertelli

15. Gaviño Rodríguez, V. (2020). Actitudes ante la lengua castellana y su enseñanza en la prensa pedagógica argentina de finales del XIX. El tema lingüístico en El Monitor de la Educación Común. En M. Rivas Zancarrón y V. Gaviño Rodríguez (Eds.), Creencias y actitudes ante la lengua en la españa y américa de los siglos XVIII y XIX, Madrid: Iberoamericana Vervuert.

16. Hernández, B. (1878). Compendio de gramática castellana. Salta: Imprenta de La Opinión.

17. Koerner, E. F. K. (2007). La historiografía de la lingüística. Pasado, presente y futuro. En Dorta, J., Corrales, C. \& Corbella, D. (Eds.), Historiografía de la lingüística en el ámbito hispánico. Fundamentos epistemológicos y metodológicos (pp. 15-56). Madrid: Arco Libros.

18. La Gaceta de Tucumán (1968). Personalidades en el olvido. Rita Pérez de Bertelli. La Gaceta de Tucumán, 1 de abril de 1968. x.

19. La Gaceta de Tucumán (1982). Rita P. de Bertelli. La Gaceta de Tucumán, 18 de octubre de 1982. X.

20. Lacanna, G. (2011). Tradición gramatical y concepciones lingüísticas en Idioma patrio. Una gramática elemental (1910), de Z. Vélez de Aragón. Revista Argentina de Historiografía Lingüística, 3(2), 87-108.

21. Lidgett, E. \& Toscano y García, G. (2020). La introducción de la gramática histórica y comparada en la tradición escolar argentina: un estudio de la Gramática de L. J. Bode (1858). Confluencias, 58(1), 33-57.

22. Lidgett, E. (2011). Gramática escolar y políticas lingüísticas en la Argentina: un análisis de la Gramática de la lengua castellana (1893) de Baldmar Dobranich y Ricardo Monner Sans. En Revista Argentina de Historiografía Lingüística, 3(2), 109-132.

23. Lidgett, E. (2017a). La consolidación de un modelo gramatical escolar en la enseñanza secundaria Argentina (1863-1936). Boletín de Filología, 52(2), 119-145.

24. Lidgett, E. (2017b). Definiciones y clasificaciones de la oración en el corpus de la gramática escolar argentina (1863-1922). Beiträge zur Geschichte der Sprachwissenschaft, 27(1), 43-72.

25. Lidgett, E. (2018). Una gramática escolar filosófica: reflexiones sobre el Curso gradual de gramática castellana de Isaac Larraín (1881). Boletín de la Sociedad Española de Historiografía Lingüística, 12, 123-153.

26. Liernur, P. (1994). El nido en la tempestad. La formación de la casa moderna en la Argentina a través de manuales y artículos de economía doméstica (1870-1930). Buenos Aires: Instituto de Arte Americano e Investigaciones Estéticas.

27. López García, M. (2009). Discusión sobre la lengua nacional en Argentina: posiciones en el debate y repercusiones en la actualidad. Revista de Investigación Lingüística, 12, 375-397.

28. Lyford, C. A. (1914). Veinte lecciones de economía doméstica. (Trad. de Rita P. de Bertelli). Tucumán: Universidad de Tucumán.

29. Lyford, C. A. (1919). Veinte lecciones de economía doméstica. (Trad. de Rita P. de Bertelli). El Monitor de la Educación Común, 37(553), 3-23.

30. Martí, G. (1876). Gramática castellana destinada al uso de los maestros que se dedican a la enseñanza de esta materia de las escuelas españolas e hispano-americanas. Buenos Aires: Librería y Papelería La Publicidad. 
Hacia la ciencia del lenguaje. Ejercicios de análisis gramatical (1916), de Rita Pérez de Bertelli

31. Morgade, G. (1993). ¿Quiénes fueron las primeras maestras?. Revista del Instituto de Ciencias de la Educación, 12, 52-60.

32. Pérez de Bertelli, R. (1915). Clase de puericultura en 3er. año. El Monitor de la Educación Común, 33(507), 503-506.

33. Pérez de Bertelli, R. (1916a). Acción educativa de Amadeo Jacques en Tucumán. Álbum general de la provincia de Tucumán en el primer centenario de la independencia argentina. Buenos Aires: Talleres Gráficos Rodríguez Giles, 1916. s.p..

34. Pérez de Bertelli, R. (1916b). Evocación. La Gaceta de Tucumán. 9 de julio de 1916. 7.

35. Pérez de Bertelli, R. (1916c). Hacia la ciencia del lenguaje. Ejercicios de análisis gramatical.

36. Pérez de Bertelli, R. (1924). Por la salud del niño: guía práctica de puericultura dedicada a las niñas de todas las clases sociales. Tucumán: Universidad Nacional de Tucumán, Liga Sanitaria del Norte Argentino.

37. Pérez de Bertelli, R. (1938). Nobles palabras. Fragmentos de un discurso. Ideas. Revista Quincenal de Educación de Tucumán II, 23. 31 de julio de 1938.

38. Pujolle, A. (1867). Gramática española. Parte teórica. Buenos Aires: Imprenta y Librería de Mayo.

39. Sastre, M. (1857). Lecciones de gramática para la enseñanza primaria. Buenos Aires: P. Morta.

40. Swiggers, P. (2009). La historiografía de la lingüística: apuntes y reflexiones. Revista Argentina de Historiografía Lingüística, 1(1), 67-76.

41. Swiggers, P. (2012). Historiografía de la gramaticografía didáctica: apuntes metodológicos con referencia a la (historia de la) gramática española y francesa. En Vila, N. (Ed.), Lengua, literatura y educación en la España del siglo $X X$ (pp. 15-38). Berna: Peter Lang / Lleida: Universitat de Lleida.

42. Tacconi de Gómez, M. (2006). Boletín de la Academia Argentina de Letras LXXI, 285-286. 367-375.

43. Toscano y García, G. (2012). Una gramática de la nación argentina. Sobre El libro del idioma, de Pedro Henríquez Ureña y Narciso Binayán. Revista Argentina de Historiografía Lingüística, 4(2), 155-165.

44. Toscano y García, G. \& García Folgado, M. J. (2012). Los textos de gramática en la escuela argentina (18631900). Escribas. Revista de Letras, 7, 27-54.

45. Toscano y García, G. \& García Folgado, M. J. (2015). La lengua y los maestros. Las «Gramáticas» de Gregorio Martí (1876 y 1877). Beiträge zur Geschichte der Sprachwissenschaft, 25(2), 221-246.

46. Toscano y García, G. \& García Folgado, M. J. (2017). La enseñanza de la sintaxis en la gramática de René Bastianini. Bulletin of Hispanic Studies, 94(6), 557-574.

47. Toscano y García, G., Lidgett, E. \& García Folgado, M. J. (2019). Sobre la Bibliografía de la gramática escolar argentina [BIGEA] (1810-1922). En Sociedad Española de Historiografía Lingüística: XII Congreso Internacional de la Sociedad Española de Historiografía Lingüística. Universidad de Bolonia: Bolonia, Italia.

48. Trombetta, A. M. (1998). Alcances y dimensiones de la educación superior no universitaria en la Argentina. Buenos Aires: Centro de Estudios de Desarrollo y Sociedad (Tesis de maestría). Mimeo.

49. Wernicke, R. (1867). El pensamiento: base de la gramática. Método gramatical, para enseñar y aprender a expresar el discípulo sus pensamientos. Buenos Aires: Imprenta Alemana. 